OPEN ACCESS

Check for updates

For numbered affiliations see end of the article.

Correspondence to: $\mathrm{H}$ Song huan@hi.is

(or @CPHIceland on Twitter; ORCID 0000-0003-3845-8079)

Additional material is published online only. To view please visit the journal online.

Cite this as: $B M J$ 2019;367:15784 http://dx.doi.org/10.1136/bmi.l5784

Accepted: 10 September 2019

\title{
Stress related disorders and subsequent risk of life threatening infections: population based sibling controlled cohort study
}

\author{
Huan Song, 1,2,3 Katja Fall, 2,4 Fang Fang, ${ }^{2}$ Helga Erlendsdóttir, ${ }^{5,6}$ Donghao Lu, ${ }^{2}$ \\ David Mataix-Cols, ${ }^{7,8}$ Lorena Fernández de la Cruz, ${ }^{7,8}$ Brian M D’Onofrio, ${ }^{2,9}$ Paul Lichtenstein, ${ }^{2}$ \\ Magnús Gottfreðsson, ${ }^{5,10}$ Catarina Almqvist, ${ }^{2,11}$ Unnur A Valdimarsdóttir ${ }^{1,2,12}$
}

\section{ABSTRACT}

OBJECTIVE

To assess whether severe psychiatric reactions to trauma and other adversities are associated with subsequent risk of life threatening infections.

\section{DESIGN}

Population and sibling matched cohort study.

SETTING

Swedish population.

PARTICIPANTS

144919 individuals with stress related disorders (post-traumatic stress disorder (PTSD), acute stress reaction, adjustment disorder, and other stress reactions) identified from 1987 to 2013 compared with 184612 full siblings of individuals with a diagnosed stress related disorder and 1449190 matched individuals without such a diagnosis from the general population.

\section{MAIN OUTCOME MEASURES}

A first inpatient or outpatient visit with a primary diagnosis of severe infections with high mortality rates (ie, sepsis, endocarditis, and meningitis or other central nervous system infections) from the Swedish National Patient Register, and deaths from these infections or infections of any origin from the Cause of Death Register. After controlling for multiple confounders, Cox models were used to estimate hazard ratios of these life threatening infections.

RESULTS

The average age at diagnosis of a stress related disorder was 37 years (55 541, 38.3\% men). During a mean follow-up of eight years, the incidence of life

\section{WHAT IS ALREADY KNOWN ON THIS TOPIC}

Psychological stress might increase susceptibility to infections through compromised immunity

A series of experimental studies on humans and other animals suggests a link between psychological stress and acute respiratory tract infections

Data are, however, limited on more severe life threatening infections such as meningitis and sepsis

\section{WHAT THIS STUDY ADDS}

This study found an association between stress related disorders and risk of life threatening infections, including sepsis, endocarditis, central nervous system infections, and fatal infections of any other origin

The association was more pronounced among people with a diagnosed stress related disorder at a younger age and those with psychiatric comorbidities

The long term use of selective serotonin reuptake inhibitors was associated with an attenuated risk of life threatening infections after diagnosis of a stress related disorder

threatening infections per 1000 person years was 2.9 in individuals with a stress related disorder, 1.7 in siblings without a diagnosis, and 1.3 in matched individuals without a diagnosis. Compared with full siblings without a diagnosis of a stress related disorder, individuals with such a diagnosis were at increased risk of life threatening infections (hazard ratio for any stress related disorder was 1.47 (95\% confidence intervals1.37 to 1.58) and for PTSD was 1.92 (1.46 to 2.52$)$ ). Corresponding estimates in the population based analysis were similar (1.58 (1.51 to 1.65 ) for any stress related disorder, $\mathrm{P}=0.09$ for difference between sibling and population based comparison, and 1.95 (1.66 to 2.28) for PTSD, $\mathrm{P}=0.92$ for difference). Stress related disorders were associated with all studied life threatening infections, with the highest relative risk observed for meningitis (sibling based analysis 1.63 (1.23 to 2.16)) and endocarditis (1.57 (1.08 to 2.30)). Younger age at diagnosis of a stress related disorder and the presence of psychiatric comorbidity, especially substance use disorders, were associated with higher hazard ratios, whereas use of selective serotonin reuptake inhibitors in the first year after diagnosis of a stress related disorder was associated with attenuated hazard ratios.

\section{CONCLUSION}

In the Swedish population, stress related disorders were associated with a subsequent risk of life threatening infections, after controlling for familial background and physical or psychiatric comorbidities.

\section{Introduction}

Excessive or prolonged psychological stress compromises several physiological systems, which might increase susceptibility to disease. ${ }^{1}$ Strong evidence from animal models ${ }^{2}$ and human studies ${ }^{13}$ suggests a considerable modulation of the hypothalamicpituitary-adrenal axis in response to stress, with altered biological functions such as compromised immunity (eg, impaired humoral and cell mediated immunity $)^{1}$ and increased inflammatory reactivity. ${ }^{1}$ Correspondingly, people exposed to psychological stress have been reported to have a higher risk of respiratory virus infections $s^{4-6}$ paralleled with reduced immune responses to several antiviral and antibacterial vaccines. $^{7-10}$

Stress related disorders, including post-traumatic stress disorder (PTSD), acute stress reaction (also known as acute stress disorder), adjustment disorder, and other stress reactions, refer to a group of psychiatric conditions that are preceded and triggered by 
an identifiable trauma or other life stressors. ${ }^{11}$ With considerable variation in response to adverse events, people with stress related disorders might represent a population with the most severe physiological dysregulation as a result of severe stress. ${ }^{1}$ Indeed, disrupted immune profiles have been reported in populations with PTSD and other stress related disorders ${ }^{12-14}$ as well as an increased risk of various autoimmune diseases. ${ }^{15}$ Recent data suggest an association between PTSD and a number of infectious diseases, ${ }^{16}$ although data are as yet scarce on the role of stress-related disorder in major life threatening infections. Taking advantage of nationwide registers in Sweden, with complete information on medical diagnoses and family links, we conducted a population based sibling controlled cohort study to explore the association between stress related disorders and risk of life threatening infections.

\section{Methods \\ Study design}

From the Swedish National Patient Register we identified all Sweden born residents with a first diagnosis of a stress related disorder between 1 January 1987 and 31 December 2013 ( $n=156537)$. This register contains nationwide data on inpatient care from 1987 and specialist outpatient care from 2001. Utilising the national identification numbers that are uniquely assigned to all Swedish residents, we linked the cohort with stress related disorders to other health registers in Sweden.

We excluded those with a diagnosis of a stress related disorder at age 5 or younger $(\mathrm{n}=139),{ }^{17}$ a history of any life threatening infection before the diagnosis of a stress related disorder $(n=4311)$, conflicting information (died or emigrated before the diagnosis, $\mathrm{n}=24$ ), or missing information on county of birth $(n=21)$. To ensure complete family links from the Swedish Multi-Generation Register, ${ }^{18}$ we further excluded 7123 people born before 1932, leaving 144919 people for analysis. The date of diagnosis was the index date.

\section{Sibling cohort}

To control for familial confounding, ${ }^{17}$ we constructed a sibling cohort to compare individuals with a stress related disorder with their unaffected full siblings. Through the Multi-Generation Register, we identified 184612 full siblings (of 103072 (71.1\%) individuals with a stress related disorder) without a diagnosis of a stress related disorder or life threatening infection at the diagnosis date of the affected sibling (ie, index date).

\section{Population matched cohort}

A matched cohort design was used to compare individuals with a stress related disorder with the general population. For each individual with a stress related disorder diagnosis, we then randomly selected 10 people from the Total Population Register with no diagnosis of a stress related disorder or life threatening infection at the diagnosis date of the affected individual (ie, index date). We individually matched individuals without a diagnosis of stress related disorder to individuals with such a diagnosis by sex, birth year, and county of birth.

\section{Follow-up}

Follow-up of the study cohorts started from the index date until the occurrence of any or a specific type of life threatening infection, death, emigration, or the end of follow-up (31 December 2013), whichever occurred first. The follow-up for unaffected full siblings or individuals without a diagnosis of stress related disorder was additionally censored if a stress related disorder was diagnosed after the index date.

\section{Stress related disorders}

We defined stress related disorders as any first inpatient or outpatient visit with the main diagnosis of a stress related disorder registered in the National Patient Register according to ICD-9 (international classification of diseases, ninth revision) codes 308 or 309 and ICD-10 (10th revision) code F43. Stress related disorders were further divided into PTSD (ICD9: 309B; ICD-10: F43.1), acute stress reaction (ICD-9: 308, 309A; ICD-10: F43.0), and adjustment disorder and other stress reactions (ICD-9: 309X; ICD-10: F43.8, F43.9, see supplementary table 1). Because PTSD might initially be diagnosed as other stress related disorders (eg, acute stress reaction ${ }^{19}$ ), we considered individuals with a PTSD diagnosis within one year after their first stress related disorder diagnosis to have had a PTSD diagnosis from the date of first diagnosed stress related disorder.

From the Swedish Prescribed Drug Register (July 2005 onwards) we obtained information on prescribing of selective serotonin reuptake inhibitors (Anatomical Therapeutic Chemical code N06AB) within the first year after the diagnosis of a stress related disorder. Although the use of selective serotonin reuptake inhibitors in children and young people is debatable, ${ }^{20}$ this drug class has been widely used ${ }^{21}$ and recommended as the first line pharmacotherapy for adults with stress related disorders (for example, in Sweden, ${ }^{22}$ United Kingdom, ${ }^{23}$ and United States ${ }^{24}$ ). We defined users of selective serotonin reuptake inhibitors as those with two or more dispensations of this drug. We calculated the average dosage by dividing the cumulative defined daily dose by the interval (days) from the first to the last dispensation; and this interval was also considered as the length of selective serotonin reuptake inhibitors treatment.

\section{Life threatening infections}

We identified incident cases of severe infections characterised by high fatality (sepsis, endocarditis, and meningitis or other central nervous system infections) as any first inpatient or outpatient visit with these infections as the main diagnosis, or death with these infections as the underlying cause of death from the Cause of Death Register. In addition, we identified 
all lethal infections of any other origin by identifying deaths with other infections documented as the underlying cause of death from the Cause of Death Register (see supplementary table 1).

\section{Covariates}

Data on education level, family income, and marital status were obtained from the Longitudinal Integration Database for Health Insurance and Labour Market study database. Other psychiatric disorders are commonly diagnosed around the same time as diagnosis of stress related disorders. ${ }^{25} 26$ Given that co-occurring psychiatric disorders might also be related to the trauma preceding the diagnosis of stress related disorder, and as such represent more severe stress reactions, we considered other psychiatric diagnoses from three months before to one year after the diagnosis of stress related disorder as psychiatric comorbidity. In contrast, we considered other psychiatric disorders documented more than three months before the diagnosis of a stress related disorder as indicating a history of other psychiatric disorders. We further obtained information on history of severe somatic diseases (myocardial infarction, congestive heart failure, cerebrovascular disease, chronic pulmonary disease, connective tissue disease, diabetes, renal diseases, liver diseases, ulcer diseases, and HIV infection/AIDS), ${ }^{27}$ and history of inpatient visit because of any infectious disease (as an indicator of baseline susceptibility to infectious diseases). The diagnoses were obtained from the National Patient Register, with corresponding ICD codes (see supplementary table 1). Family history of major life threatening infections was defined as any diagnosis of or death due to sepsis, endocarditis, and meningitis or other central nervous system infections among biological parents and full siblings of the study individuals, according to the National Patient Register or the Cause of Death Register. Except for the categories of history of other psychiatric disorder and of psychiatric comorbidity, we updated information until the index date (baseline) for all other covariates. For sensitivity analyses on somatic comorbidities and behaviour related factors, data on the presence of severe somatic diseases and substance use or sleep related diseases (see supplementary table 1) after the index date were also extracted from the National Patient Register. Anatomical defects (congenital diseases of heart and nervous system) are risk factors for severe infections, ${ }^{28}$ and therefore were identified from the National Patient Register and Medical Birth Register (available from 1973 onwards).

\section{Statistical analysis}

We estimated the association between stress related disorders and risk of life threatening infections using hazard ratios with $95 \%$ confidence intervals, derived from conditional Cox regression models. Time since the index date was applied as the underlying time scale.

In the sibling cohort, all models were stratified by family identifier and adjusted for sex, birth year, education level ( $<9$ years, 9-12 years, >12 years, unknown), family income (top 20\%, middle, lowest $20 \%$, unknown), marital status (single, married or cohabiting, divorced or widowed), history of severe somatic diseases (yes or no), history of other psychiatric disorders (yes or no), and history of inpatient visit because of any infectious diseases (yes or no). We first considered stress related disorders as one group and then by diagnostic categories of PTSD, acute stress reaction, and adjustment disorder and other stress reactions. Also, in addition to a diagnosis of any life threatening infection, we separately examined the risk of sepsis, endocarditis, and meningitis or, other central nervous system infections, and deaths due to infections of any other origin.

In subgroup analyses, we calculated the hazard ratios by sex (men or women), time since index date ( $<1$ year, 1-4 years, 5-9 years, or $\geq 10$ years), history of severe somatic diseases (yes or no), calendar period at the index date (1987-2000, 2001-05, or 2006-13), history of other psychiatric disorders (yes or no), family history of major life threatening infections (yes or no), and history of inpatient visit because of any infectious diseases (yes or no). The differences of subgroup hazard ratios were assessed by introducing interaction terms to the Cox models or by computing Wald tests. In addition, to examine potential effect modification by age at index date on the interested association, we applied restricted cubic splines on age and integrated these to the Cox models by adding an interaction term. ${ }^{29}$ Age varying hazard ratios were estimated and visualised thereafter.

To study the potential impact of severity and complexity of stress related disorders on the studied associations, we assessed hazard ratios by the presence of psychiatric comorbidity (any psychiatric comorbidity, as well as by specific type, including depression, anxiety, and substance use disorders) and by the type of psychiatric care received at diagnosis (inpatient or outpatient care). Within one year after the diagnosis of a stress related disorder, we considered the psychiatric comorbidity as a time varying variable.

We repeated the main analyses in the population based cohort, where we used conditional Cox models stratified by matching identifiers (sex, birth year, and county of birth), adjusting for family history of major life threatening infections (yes or no) and all other covariates. We compared hazard ratios between sibling and population based analyses using a $\mathrm{z}$ test. ${ }^{30}$ Furthermore, we restricted analyses to individuals with a diagnosis of a stress related disorder after July 2005 and with more than one year of follow-up, and compared the risk of life threatening infections beyond one year between subgroups with different status of selective serotonin reuptake inhibitors use.

To test the robustness of the observed associations, we performed several sensitivity analyses. To rule out the possibility that the observed risk increase was due to a pre-existing or co-occurring medical condition, we excluded from the analysis individuals with any diagnosis of severe somatic diseases, injuries and poisonings, or infectious diseases (see codes in 
supplementary table 1 ) within one year before the index date. In addition, to alleviate concerns that the observed associations were accounted for by the poorer health conditions or suboptimal behaviours of individuals with stress related disorders than those without, we restricted our analyses to individuals without a history of severe somatic diseases and additionally adjusted the Cox models by the presence of severe somatic conditions (as time varying variables) or substance use or sleep related diagnoses (as a binary variable) during follow-up. Lastly, to account for increased risk of infection from anatomical defects, we repeated our analyses after excluding those with congenital heart or nervous system diseases. Analyses were conducted in SAS statistical software, version 9.4 (Cary, NC) and STATA 15 (StataCorp).

\section{Patient and public involvement}

No patients were involved in proposing the research question or the outcome measures, nor were they involved in developing plans for design or implementation of the study. There are no plans to disseminate the results of the research directly to study participants or the relevant patient community. Dissemination to the Swedish population (which constitutes the study population) will be through media outreach (eg, press release and communication) on publication of this study.

\section{Results}

Fig 1 shows the flow chart of identifying study cohorts through the study. The sibling cohort accrued 2370354 person years, with on average eight years of follow-up. The mean age at entry to the study was 37 years (table 1), and 38.3\% (39513/103072) of the individuals with a diagnosed stress related disorder were men. A history of other psychiatric disorders, severe somatic diseases, and inpatient stay for infectious diseases were more common among individuals with a diagnosis of a stress related disorder than among unaffected full siblings $(34.8 \% v 12.6 \%$, $16.5 \% \vee 12.8 \%$, and $30.9 \% \vee 23.8 \%$, respectively). In addition, individuals with a diagnosis tended to have lower family income and were more likely to be divorced or widowed (table 1).

During follow-up, 4843 individuals with incident life threatening infections were identified (2197 among individuals with a diagnosis of a stress related disorder and 2646 among unaffected full siblings, with a crude incidence rate of 2.7 and 1.7 per 1000 person years, respectively). After controlling for all covariates, an association was observed between stress related disorders and life threatening infections: hazard ratios were 1.47 (95\% confidence interval 1.37 to 1.58) for any stress related disorder, 1.92 (1.46 to 2.52 ) for PTSD (fig 2), 1.43 (1.29 to 1.58) for acute stress reaction, and 1.48 (1.33 to 1.64) for adjustment disorder and other stress reactions (see supplementary fig 1). Stress related disorders were associated with all studied life threatening infections, with hazard ratios varying from 1.39 (1.16 to 1.65 ) for deaths due to infections of other origin to 1.63 (1.23 to 2.16) for meningitis. The population based comparisons corroborated these associations (fig 2 and supplementary fig 1) as differences between the estimates in the sibling based and population based analyses were not significant: hazard ratio for any stress related disorder was 1.58 (1.51 to 1.65 ), $\mathrm{P}=0.09$ for difference between within sibling and population based comparison; hazard ratio for PTSD was 1.95 (1.66 to 2.28), $\mathrm{P}=0.92$ for difference.

Based on both sibling and population based analyses, the observed associations did not differ by sex, calendar period, family history of life threatening infections, or history of inpatient stay for infectious disease (table 2 and supplementary table 2). Associations seemed stronger, however, among individuals without a history of severe somatic diseases or of other psychiatric disorders both $(\mathrm{P}<0.001$ for interaction in population based analysis) and within the first year after the diagnosis of a stress related disorder ( $\mathrm{P}<0.001$ for difference in population based analysis). Moreover, an age dependent risk pattern suggested a linear decline in hazard ratios with increased age at diagnosis (fig 3).

Additionally, hazard ratios were higher for any stress related disorder diagnosed through inpatient hospital care than through outpatient specialist care (supplementary table 3, $\mathrm{P}=0.009$ for difference in population based analysis). For patients with stress related disorders other than PTSD, the presence of psychiatric comorbidity, especially comorbid substance use disorders, was linked to a further increased relative risk of life threatening infections in both sibling and population based analyses (supplementary fig 2).

Among individuals with a diagnosis of a stress related disorder after July $2005(n=74691)$, the use of selective serotonin reuptake inhibitors after diagnosis was associated with a lower risk of life threatening infections beyond one year (users compared with non-users: hazard ratio 0.81 ( 0.66 to 0.98 ), $\mathrm{P}=0.03$ ). Persistent use of selective serotonin reuptake inhibitors in the first year after diagnosis of a stress related disorder was associated with a linear attenuation in the relative risk of subsequent life threatening infections (hazard ratio 0.96 (0.66 to1.40), 0.85 (0.64 to 1.13), and 0.70 (0.52 to0.94) for 179 or less, 180-319, and 320 days or more of use, respectively, $\mathrm{P}=0.01$ for trend; supplementary table 4).

Restricting the analyses to individuals without a diagnosis of severe somatic diseases, injury, or infectious diseases within one year before the index date, or those without anatomical defects, yielded largely identical results as the main analyses (supplementary tables 5 and 6). Moreover, although additional adjustments for severe somatic diseases during follow-up did not substantially modify the estimates, the hazard ratios, especially those from the population based analyses, were attenuated after additionally adjusting for the presence of substance use or sleep related diagnoses during follow-up (supplementary table 7). 


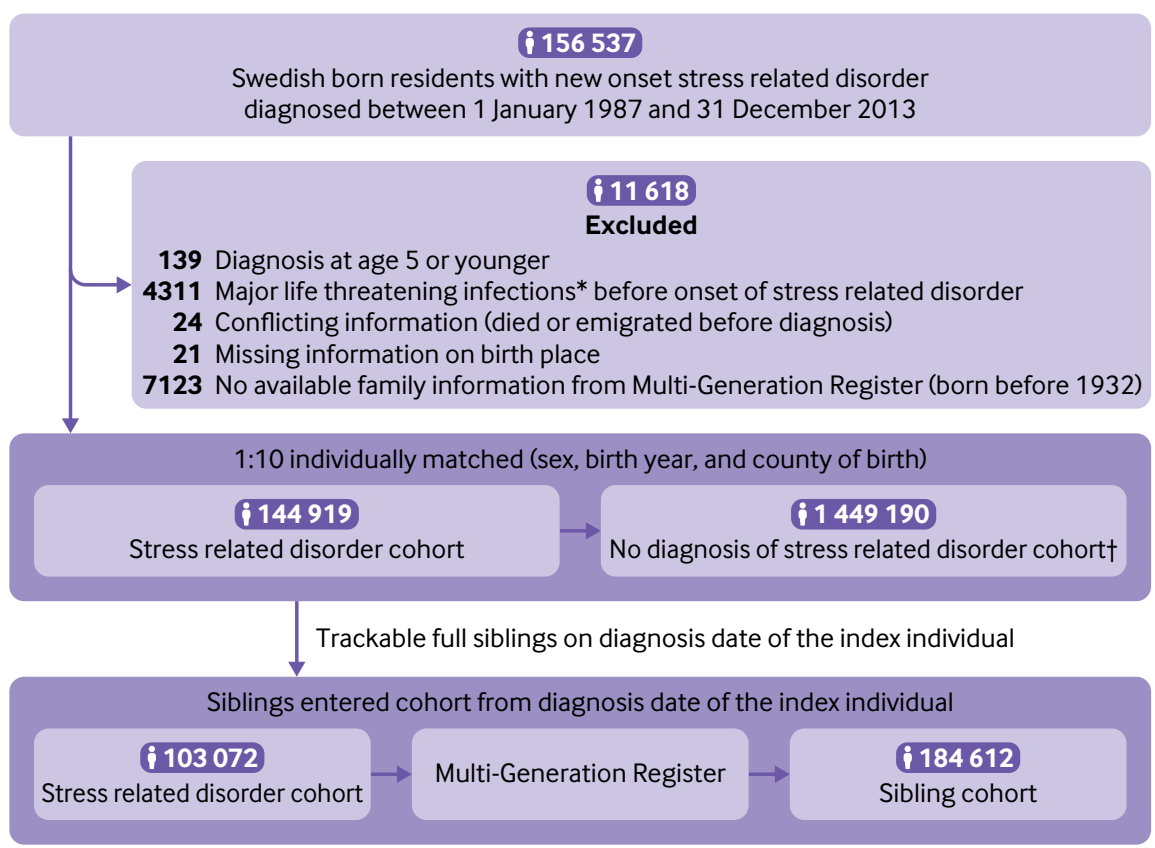

Fig 1 | Study design. *Sepsis, endocarditis, and meningitis or other central nervous system infections. tRandomly selected from general population: individuals without a diagnosis of stress related disorder or major life threatening infection at diagnosis date of the index individual

\section{Discussion}

In this study, individuals with stress related disorders, particularly when young at diagnosis, were at increased risk of life threatening infections, after taking into account sex, familial background, and baseline physical or psychiatric conditions. Psychiatric comorbidities, especially substance use disorders, were associated with further risk increases, whereas the long term ( $>1$ year) risk of life threatening infections seemed attenuated by persistent use of selective serotonin reuptake inhibitors in the first year after diagnosis of a stress related disorder.

\section{Strengths and weaknesses of this study}

The major strength of our study was the use of a large population based cohort with complete followup for up to 27 years and the comparison within full siblings to tackle the a priori concern about familial confounding. ${ }^{17}$ Information bias was minimised because the diagnosis and registration of diagnosis and outcome were compiled prospectively and independently. Also, because most of the outcomes of interest (eg, sepsis, meningitis) are aggressive diseases, characterised by sudden onset and severe symptoms, the influence of any surveillance bias or delayed diagnosis should be minor. Furthermore, the large sample size provided enough statistical power for detailed subgroup analyses; and the availability of rich sociodemographic and medical information enabled consideration of a wide range of important confounding and mediating factors.

This study has several limitations. Firstly, the late establishment of the Swedish Outpatient Register (2001) could potentially lead to underestimation of people with stress related disorders, especially milder forms. Also, changes in the definition and diagnostic criteria of stress related disorders over the study period could have influenced the observed associations. For instance, in 2005 exhaustion disorder was introduced to the Swedish ICD-10 system, which resulted in a small difference between the Swedish and international ICD10 code category F43. Similar results were, however, obtained from a subanalysis of different calendar periods, suggesting a minor influence of these factors. Secondly, we had limited information on some important behaviour related factors (eg, smoking, drug and alcohol use), and our sensitivity analyses showed a considerable mediating effect of these factors on the observed associations. Further research with detailed data on lifestyle is warranted. Thirdly, although trauma focused psychotherapy has been given the highest priority for PTSD treatment in many countries, including Sweden, ${ }^{22}$ we have no such data available for analyses. Future well designed studies are needed for exploring the influence of psychotherapy, alone or with drug treatment, on the association between stress related disorder and risk of severe infections. Fourthly, although the association between stress related disorders and risk of fatal infections seems robust, the observational nature of our analysis limits inference of causality or exploration of potential mediating pathways. For instance, despite efforts to control for susceptibility to diseases (eg, history of severe somatic diseases, other psychiatric disorders, or inpatient visit for any infectious diseases) that differ between individuals with a diagnosis of a stress related disorder and reference groups at baseline, we cannot refute the possibility that unmeasured factors contribute to the 


\begin{tabular}{|c|c|c|c|c|}
\hline \multirow[b]{2}{*}{ Characteristics } & \multicolumn{2}{|c|}{ Sibling cohort } & \multicolumn{2}{|c|}{ Population based cohort } \\
\hline & $\begin{array}{l}\text { Stress related disorder } \\
(n=103072)\end{array}$ & $\begin{array}{l}\text { Sibling } \\
(\mathrm{n}=184612)\end{array}$ & $\begin{array}{l}\text { Stress related disorder } \\
(\mathrm{n}=144919)\end{array}$ & $\begin{array}{l}\text { Matched* } \\
(n=1449190)\end{array}$ \\
\hline Mean (SD) age at index date (years) & $37.0(13.9)$ & $38.0(15.1)$ & $37.2(14.3)$ & $37.2(14.3)$ \\
\hline Mean (SD) follow-up (years) & $7.8(6.4)$ & $8.5(6.8)$ & $7.9(6.5)$ & $8.1(6.6)$ \\
\hline Men & $39513(38.3)$ & $94163(51.0)$ & $55541(38.3)$ & $555410(38.3)$ \\
\hline \multicolumn{5}{|l|}{ Education level (years): } \\
\hline$<9$ & $4191(4.1)$ & $11919(6.5)$ & $6453(4.4)$ & $58565(4.0)$ \\
\hline $9-12$ & $73505(71.3)$ & $126305(68.4)$ & $103252(71.3)$ & $941393(65.0)$ \\
\hline$>12$ & $23839(23.1)$ & $41569(22.5)$ & $32625(22.5)$ & $426442(29.4)$ \\
\hline Unknown & $1537(1.5)$ & $4819(2.6)$ & $2589(1.8)$ & $22790(1.6)$ \\
\hline \multicolumn{5}{|l|}{ Yearly family income level: } \\
\hline Lowest 20\% & $22941(22.3)$ & $33782(18.3)$ & $32847(22.7)$ & $247467(17.1)$ \\
\hline Middle & $56877(55.2)$ & $95927(52.0)$ & $79051(54.6)$ & $799409(55.2)$ \\
\hline Top 20\% & $13160(12.8)$ & $29946(16.2)$ & $18292(12.6)$ & $254009(17.5)$ \\
\hline Unknown & $10094(9.8)$ & $24957(13.5)$ & $14729(10.2)$ & $148305(10.2)$ \\
\hline \multicolumn{5}{|l|}{ Marital status: } \\
\hline Single & $58791(57.0)$ & $100525(54.5)$ & $82425(56.9)$ & $823667(56.8)$ \\
\hline Married or cohabiting & $30730(29.8)$ & $66694(36.1)$ & $42868(29.6)$ & $514251(35.5)$ \\
\hline Divorced or widowed & $13551(13.2)$ & $17393(9.4)$ & $19626(13.5)$ & $111272(7.7)$ \\
\hline \multicolumn{5}{|l|}{ History of severe somatic diseasest: } \\
\hline Yes & $17020(16.5)$ & $23534(12.8)$ & $24004(16.6)$ & $145619(10.1)$ \\
\hline No & $86052(83.5)$ & $161078(87.3)$ & $120915(83.4)$ & $1303571(90.0)$ \\
\hline \multicolumn{5}{|l|}{ History of other psychiatric disordersł: } \\
\hline Yes & $36202(34.8)$ & $23466(12.6)$ & $51905(35.8)$ & $118910(8.2)$ \\
\hline No & $67860(65.2)$ & $162605(87.4)$ & $93014(64.2)$ & $1330280(91.8)$ \\
\hline \multicolumn{5}{|l|}{ Family history of major life threatening infections: } \\
\hline Yes & $10992(10.7)$ & $20455(11.1)$ & $15548(10.7)$ & $134214(9.3)$ \\
\hline No & $92080(89.3)$ & $164157(88.9)$ & $129371(89.3)$ & $1314976(90.7)$ \\
\hline \multicolumn{5}{|l|}{ History of inpatient visit for infectious diseases: } \\
\hline Yes & $31836(30.9)$ & $43956(23.8)$ & 46269 (31.9) & $307370(21.2)$ \\
\hline No & $71236(69.1)$ & $140656(76.2)$ & $98750(68.1)$ & $1141820(78.8)$ \\
\hline \multicolumn{5}{|l|}{ Type of stress related disorders: } \\
\hline Post-traumatic stress disorder & $8105(7.8)$ & - & $11541(7.9)$ & - \\
\hline Acute stress reaction & $47195(45.8)$ & - & $66758(46.1)$ & - \\
\hline Adjustment disorder and other stress reaction & $47772(46.4)$ & - & $66620(46.0)$ & - \\
\hline \multicolumn{5}{|l|}{ Type of psychiatric care received at diagnosis: } \\
\hline Inpatient & $37352(36.2)$ & - & $52817(36.5)$ & - \\
\hline Outpatient & $65720(63.8)$ & - & $92102(63.5)$ & - \\
\hline \multicolumn{5}{|l|}{ Psychiatric comorbidity§: } \\
\hline \multicolumn{5}{|l|}{ Any } \\
\hline Yes & $22619(21.9)$ & - & $31415(21.7)$ & - \\
\hline No & $80453(78.1)$ & - & $113504(78.3)$ & - \\
\hline \multicolumn{5}{|l|}{ Depression: } \\
\hline Yes & $10581(10.3)$ & - & $14500(10.0)$ & - \\
\hline No & $92491(89.7)$ & - & $130419(90.0)$ & - \\
\hline \multicolumn{5}{|l|}{ Anxiety: } \\
\hline Yes & $6683(6.5)$ & - & $9222(6.4)$ & - \\
\hline No & 96389 (93.5) & - & $135697(93.6)$ & - \\
\hline \multicolumn{5}{|l|}{ Substance use disorder: } \\
\hline Yes & $4567(4.4)$ & - & $6514(4.5)$ & - \\
\hline No & $98505(95.6)$ & - & $138405(95.5)$ & - \\
\hline
\end{tabular}

${ }^{*}$ Matched individuals without a diagnosis of stress related disorder.

†Myocardial infarction, congestive heart failure, cerebrovascular disease, chronic pulmonary disease, connective tissue disease, dementia, diabetes, renal diseases, liver diseases, ulcer diseases, and HIV infection/AIDS

\#First diagnosis of a psychiatric disorder, other than stress related disorders, occurring more than three months before index date (ie, diagnosis date for individuals with stress related disorder, or diagnosis date of index patient for matched individuals and siblings without a diagnosis of stress related disorder).

$\S N$ New onset psychiatric disorder, other than stress related disorders, diagnosed from three months before to one year after diagnosis of stress related disorder.

reported association. Fifthly, this study only involved individuals with a clinical diagnosis of stress related disorders through a hospital or specialist visit, thus the generalisability of our findings to those with less severe stress reaction or daily stress needs further assessment.

Comparison with other studies

With few comparable data, our results reinforce the link between stress and infection shown in experimental studies. $^{4} 53132$ In the early 1990s, a prospective, although non-randomised, study of 394 healthy volunteers who received a viral challenge (nasal drops containing a low dose of respiratory viruses) after questionnaire based psychological stress assessment found that psychological stress was associated with an increased risk of acute respiratory tract infections in a dose-response manner ${ }^{4}$; and similar conclusions were also made in subsequent research. ${ }^{5132}$ As common 


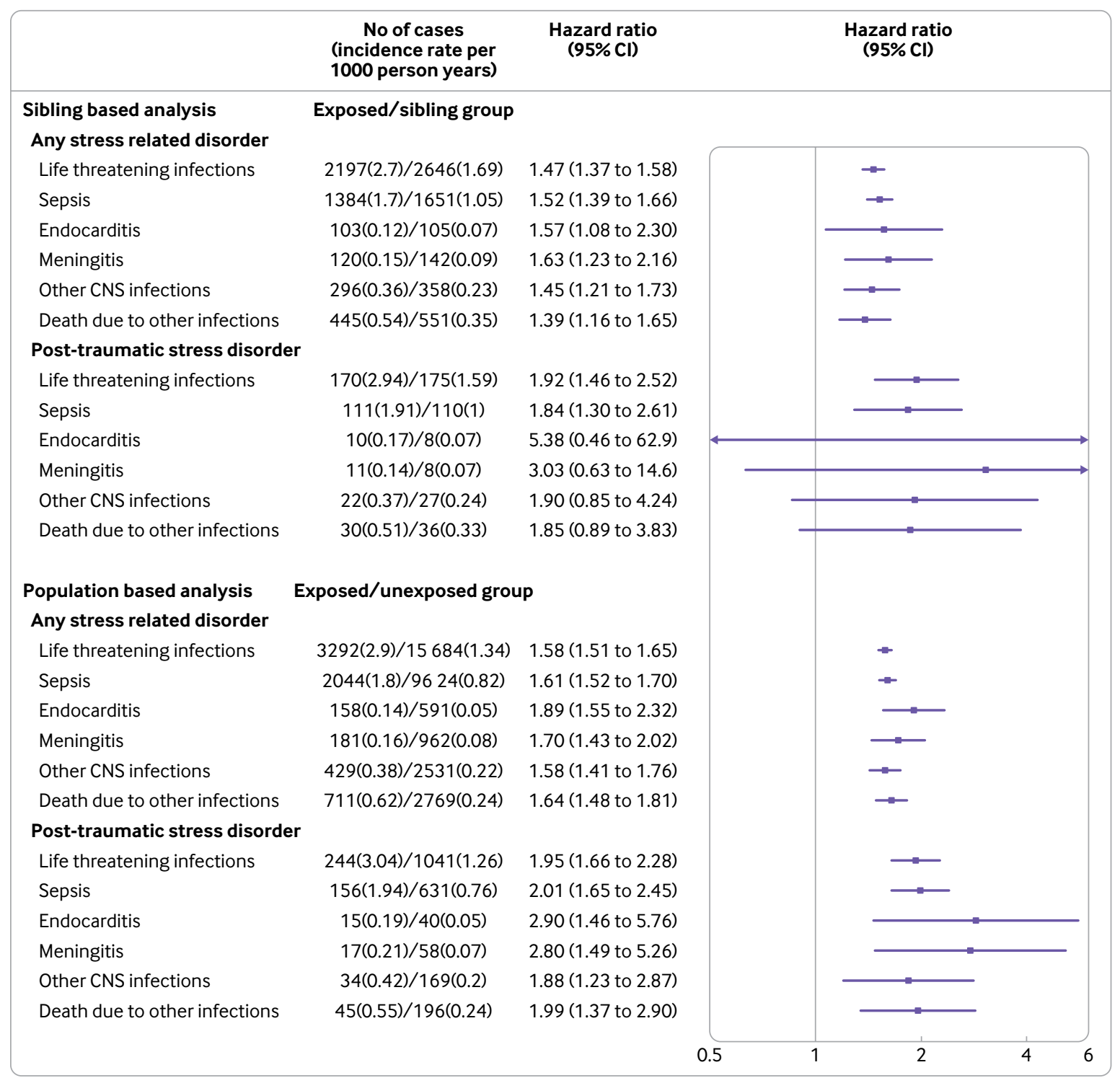

Fig 2 | Crude incidence and hazard ratios (95\% confidence intervals) for life threatening infections among individuals with any stress related disorder and post-traumatic stress disorder (exposed) compared with full siblings or matched individuals without such a diagnosis (unexposed). ${ }^{\star}$ Cox models were stratified by family identifiers and adjusted for sex, birth year, education level, family income, marital status, and history of severe somatic diseases, other psychiatric disorder, and inpatient visit for infectious diseases. + Cox models were stratified by matching identifiers (sex, birth year, and county of birth) and adjusted for education level; family income; marital status; history of severe somatic diseases, other psychiatric disorder, and inpatient visit for infectious diseases; and family history of major life threatening infections. CNS=central nervous system

respiratory viral infections were the predominant disease models in the investigations, however, it remains unclear whether the stress induced immune modulation leads to more severe infection related consequences.

Consistent with our findings, one recent Danish register based study, involving 4984 patients with PTSD diagnosed between 1995 and 2011, reported an association between PTSD and 34 types of infectious diseases, including infections of central nervous system. ${ }^{16}$ Also, one cohort study ${ }^{33}$ indicated that a higher perceived stress level was moderately associated with one year and 10 year risk of sepsis in a sample of 30183 community dwelling adults aged 45 years or older in the United States. Although these results were suggestive, these studies were either focused on PTSD only or based on one time measurement of psychological stress in an older population, with limited observed incident cases of severe infections and little control of familial and comorbidity factors.

\section{Meaning of the study}

With a specific focus on clinically diagnosed stress related disorders, we show that severe stress reactions, even transient ones (eg, acute stress reaction), were associated with an increased risk of life threatening infections, both in the short and in the long term. Importantly, the observed excess risks remained after controlling for most of the known risk factors of the studied infections, ${ }^{34-36}$ such as socioeconomic factors, 


\begin{tabular}{|c|c|c|c|c|c|c|}
\hline \multirow[b]{2}{*}{ Characteristics } & \multicolumn{3}{|c|}{$\begin{array}{l}\text { No of cases (incidence per } 1000 \text { person years): sibling } \\
\text { based analysis }\end{array}$} & \multicolumn{3}{|c|}{$\begin{array}{c}\text { No of cases (incidence per } 1000 \text { person years): } \\
\text { population based analysis }\end{array}$} \\
\hline & $\begin{array}{l}\text { Stress related } \\
\text { disorder cohort }\end{array}$ & $\begin{array}{l}\text { Sibling } \\
\text { cohort }\end{array}$ & $\begin{array}{l}\text { Hazard ratio } \\
(95 \% \mathrm{Cl})^{\star}\end{array}$ & $\begin{array}{l}\text { Stress related } \\
\text { disorder cohort }\end{array}$ & $\begin{array}{l}\text { No stress related } \\
\text { disorder cohort }\end{array}$ & $\begin{array}{l}\text { Hazard ratio } \\
(95 \% \mathrm{Cl}) \dagger\end{array}$ \\
\hline \multicolumn{7}{|l|}{ Sex: } \\
\hline Men & $983(3.11)$ & $1500(1.89)$ & $1.44(1.26$ to 1.64$)$ & $1444(3.29)$ & $7034(1.52)$ & 1.57 (1.47 to 1.67$)$ \\
\hline Women & $1214(2.44)$ & $1146(1.48)$ & 1.41 (1.24 to 1.59$)$ & $1848(2.66)$ & $8650(1.22)$ & 1.59 (1.50 to 1.68$)$ \\
\hline \multicolumn{7}{|c|}{ Time since index date (years): } \\
\hline$<1$ & $266(2.71)$ & $230(1.30)$ & 1.61 (1.30 to 2.00$)$ & $410(2.98)$ & $1438(1.04)$ & 2.04 (1.81 to 2.30$)$ \\
\hline $1-4$ & $723(2.34)$ & $805(1.42)$ & $1.53(1.36$ to 1.73$)$ & $1045(2.43)$ & $5053(1.16)$ & $1.45(1.35$ to 1.56$)$ \\
\hline $5-9$ & $543(2.45)$ & $694(1.64)$ & $1.35(1.18$ to 1.54$)$ & $811(2.64)$ & $3941(1.25)$ & $1.51(1.39$ to 1.65$)$ \\
\hline$\geq 10$ & $665(3.59)$ & $917(2.30)$ & $1.50(1.32$ to 1.70$)$ & $1026(3.99)$ & $5252(1.88)$ & $1.65(1.53$ to 1.78$)$ \\
\hline \multicolumn{7}{|c|}{ History of severe somatic diseases‡: } \\
\hline Yes & $676(5.66)$ & $663(3.92)$ & 1.37 (1.06 to 1.76$)$ & $1044(6.09)$ & $3452(3.40)$ & $1.38(1.23$ to 1.56$)$ \\
\hline No & $1521(2.19)$ & $1983(1.42)$ & 1.49 (1.37 to 1.62$)$ & $2248(2.34)$ & $12232(1.14)$ & $1.65(1.57$ to 1.73$)$ \\
\hline \multicolumn{7}{|c|}{ Calendar year at index date: } \\
\hline $1987-2000$ & $1019(2.82)$ & $1330(1.72)$ & 1.51 (1.37 to 1.67$)$ & $1564(3.10)$ & 7401 (1.39) & $1.66(1.56$ to 1.77$)$ \\
\hline 2001-05 & $696(2.52)$ & $833(1.69)$ & 1.31 (1.16 to 1.48$)$ & $1035(2.70)$ & $5183(1.34)$ & $1.45(1.34$ to 1.57$)$ \\
\hline $2006-13$ & $482(2.74)$ & $483(1.60)$ & 1.64 (1.43 to 1.88$)$ & $693(2.81)$ & $3100(1.25)$ & $1.60(1.48$ to 1.74$)$ \\
\hline \multicolumn{7}{|c|}{ History of other psychiatric disorders§: } \\
\hline Yes & $967(4.09)$ & $576(3.86)$ & $1.25(1.01$ to 1.56$)$ & $1465(4.33)$ & $2308(3.44)$ & $1.26(1.12$ to 1.41$)$ \\
\hline No & $1230(2.13)$ & $2070(1.46)$ & $1.58(1.45$ to 1.73$)$ & $1827(2.30)$ & $13376(1.21)$ & 1.79 (1.70 to 1.88$)$ \\
\hline \multicolumn{7}{|c|}{$\begin{array}{l}\text { Family history of major life threatening infec- } \\
\text { tions: }\end{array}$} \\
\hline Yes & $219(3.47)$ & $248(1.99)$ & 1.51 (1.20 to 1.89$)$ & $300(3.43)$ & $1259(1.64)$ & 1.81 (1.39 to 2.37$)$ \\
\hline No & $1978(2.64)$ & $2398(1.66)$ & $1.38(1.28$ to 1.48$)$ & $2992(2.86)$ & $14425(1.32)$ & $1.60(1.53$ to 1.67$)$ \\
\hline \multicolumn{7}{|c|}{ History of inpatient stay for infectious diseases: } \\
\hline Yes & $931(3.94)$ & $812(2.50)$ & 1.25 (1.03 to 1.50$)$ & $1405(4.14)$ & $4321(2.04)$ & $1.52(1.39$ to 1.66$)$ \\
\hline No & $1266(2.19)$ & $1834(1.48)$ & $1.58(1.44$ to 1.74$)$ & $1887(2.38)$ & $11363(1.19)$ & 1.69 (1.60 to 1.78$)$ \\
\hline \multicolumn{7}{|c|}{$\begin{array}{l}\text { *Cox models were stratified by family identifiers and adjusted for sex, birth year, education level, family income, marital status, and history of severe somatic diseases, other psychiatric disorder, } \\
\text { and inpatient visit for infectious disease. } \\
\text { +Cox models were stratified by matching identifiers (sex, birth year, and county of birth) and adjusted for education level; family income; marital status; history of severe somatic diseases, other } \\
\text { psychiatric disorder, and inpatient visit for infectious diseases; and family history of major life threatening infections. } \\
\text { fMyocardial infarction, congestive heart failure, cerebrovascular disease, chronic pulmonary disease, connective tissue disease, dementia, diabetes, renal diseases, liver diseases, ulcer diseases, } \\
\text { and HIV infection/AIDS. } \\
\text { \&First diagnosis of a psychiatric disorder, other than stress related disorders, occurring more than three months before index date. }\end{array}$} \\
\hline
\end{tabular}

familial background, physical conditions at baseline (including baseline susceptibility to infection), and the occurrence of other severe somatic diseases during follow-up. Although relatively rare, severe infections contribute substantially to the global burden of disease because of high fatality, risk of long term complications, and high healthcare costs. ${ }^{37} 38$ In contrast, stress related disorders are common in the general population. The reported lifetime prevalence of PTSD in Sweden was 5.6\% in 2005, ${ }^{39}$ and our data suggest at least a 10 times higher prevalence for other stress related disorders, underscoring the considerable clinical significance and public health implications of our findings.

Initial attempts at explaining the documented association between stress and infection were concentrated on altered circulating glucocorticoid levels and the role these played in suppression of cell mediated and humoral immunity, ${ }^{40} 41$ which could potentially increase vulnerability to infections among stressed individuals. Yet studies testing the association between glucocorticoid levels and risk of infections produced mixed results. ${ }^{42-45}$ A recent hypothesis places focus on the underlying inflammation, where glucocorticoid receptor resistance results in overproduction of inflammatory cytokines. ${ }^{646}$ This notion gains support from several studies, including the present one, implying that traumatic stress may exacerbate the severity of future infections. ${ }^{447}$

Alternative explanations for the impact of severe stress reactions on life threatening infection include behaviour related changes after the diagnosis of a stress related disorder. In the present study we observed a further increased relative risk among individuals with a diagnosis of stress related disorders and with comorbid substance use disorders, as well as attenuated excess risk after additionally adjusting for substance use and sleep related diagnoses during follow-up. It is therefore possible that behavioural factors (eg, smoking, alcohol or drug use, and sleep disturbance) at least partially mediate the observed association, through increased possibility of exposure to pathogens (eg, needle sharing among drug users ${ }^{48}$ ) or inducing immune dysfunction, or both. ${ }^{49}$ Nevertheless, it is unlikely that such behavioural factors can fully explain the increase in fatal infection related consequences, especially those that appear shortly after a diagnosis of stress related disorder.

Our finding suggesting that a diagnosis of stress related disorders in early life is associated with the largest relative risk increase in life threatening infections is in line with findings showing that childhood exposure to trauma might have a lifelong impact on susceptibility to disease, through pro- 

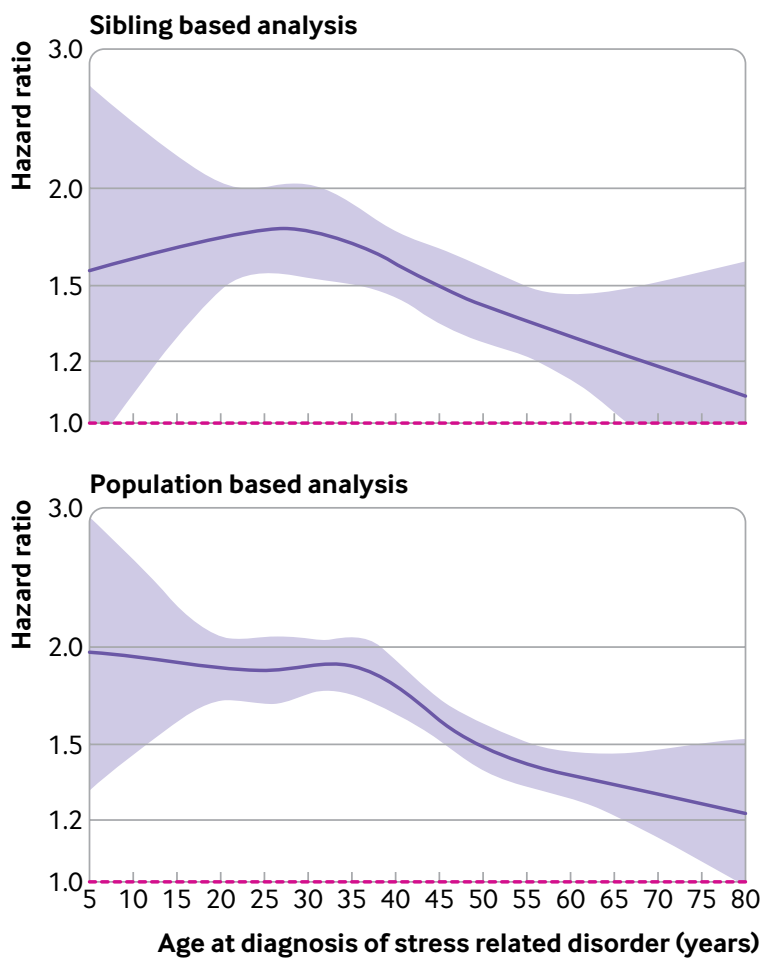

Fig 3 | Association between stress related disorders and life threatening infections by age at index date. *Restricted cubic splines were applied on age at index date, with five knots placed at $5,27.5,50,72.5$, and 95 quantiles of the distribution of outcome events. Age varying hazard ratios were then predicted based on fully adjusted Cox models where interaction terms between stress related disorders and splined age profiles were added. In sibling based analysis, the cox models were stratified by family identifiers and adjusted for sex, birth year, education level, family income, marital status, and history of severe somatic diseases, inpatient visit for infectious diseases, and other psychiatric disorder. In population based analysis, the cox models were stratified by matching identifiers-that is, sex, birth year, and county of birth, and adjusted for education level; family income; marital status; history of severe somatic diseases, inpatient visit for infectious diseases, and other psychiatric disorders; and family history of major life threatening infections. Shaded area represents $95 \%$ confidence intervals for patients with stress related disorders, especially those diagnosed at younger age. In addition, our findings, subject to replication, suggest an attenuation in the relative risk of life threatening infections with use of selective serotonin reuptake inhibitors. Further studies are needed to understand the potential mediating role of behaviour related factors in the observed association as well as potential mitigating role of various treatments for stress related disorders in reducing the excess risk of life threatening infections.

\section{AUTHOR AFFILIATIONS}

${ }^{1}$ Centre of Public Health Sciences, Faculty of Medicine, University of Iceland, Sturlugata 8, 101 Reykjavík, Iceland

${ }^{2}$ Department of Medical Epidemiology and Biostatistics, Karolinska Institutet, Stockholm, Sweden

${ }^{3}$ West China Biomedical Big Data Centre, West China Hospital, Sichuan University, Chengdu, China

${ }^{4}$ Clinical Epidemiology and Biostatistics, School of Medical Sciences, Örebro University, Örebro, Sweden

${ }^{5}$ Faculty of Medicine, University of Iceland, Reykjavík, Iceland

${ }^{6}$ Department of Clinical Microbiology, Landspítali University Hospital, Reykjavík, Iceland

${ }^{7}$ Department of Clinical Neuroscience, Centre for Psychiatry Research, Karolinska Institutet, Stockholm, Sweden

${ }^{8}$ Stockholm Health Care Services, Region Stockholm, Stockholm, Sweden

${ }^{9}$ Department of Psychological and Brain Sciences, Indiana University, Bloomington, IN, USA

${ }^{10}$ Department of Infectious Diseases, Landspítali University Hospital, Reykjavik, Iceland

${ }^{11}$ Astrid Lindgren Children's Hospital, Karolinska University Hospital, Stockholm, Sweden

${ }^{12}$ Department of Epidemiology, Harvard T H Chan School of Public Health, Boston, MA, USA

We thank Peter Green for his input on readability and accuracy of this manuscript during the review process.

Contributors: HS and UAV conceived and designed the study. HS UAV, KF, and FF analysed the data. UAV, HS, KF, FF, HE, DL, DM-C, LFC, BMD'O, PL, MG, and CA interpreted the data. HS, UAV, KF, FF, HE, DL, DM-C, LFC, BMD'O, PL, MG, and CA drafted the manuscript: HS and UAV had full access to all the data in the study and take responsibility for the integrity and accuracy of the data. HS and UAV are the guarantors. The corresponding author attests that all listed authors meet authorship criteria and that no others meeting the criteria have been omitted.

moting inflammatory reactions, ${ }^{50} 51$ interrupting neuropsychological and cognitive development, ${ }^{52} 53$ or an interplay between genes and the environment. ${ }^{54}$ Indeed, the extent of epigenetic modifications, measured as frequency of DNA methylation changes, were up to 12 times higher in participants with PTSD who were exposed to trauma in childhood compared with participants with PTSD who were trauma-free in childhood. ${ }^{54}$ These results constitute a molecular basis implying potentially more extensive biological disruptions and thereby potentially worse health outcomes for younger, rather than older, people with stress related disorders.

\section{Conclusions}

In this study, clinically diagnosed stress related disorders were associated with an increased risk of life threatening infections in the Swedish population. Despite a relatively low absolute risk, the high mortality from life threatening infections calls for increased clinical awareness among health professionals caring
Funding: The study was supported by Grant of Excellence, Icelandic Research Fund (grant No 163362-051, UAV), and European Research Council (ERC) consolidator grant (StressGene, grant No 726413 , UAV); the Karolinska Institutet (Senior Researcher Award and Strategic Research Area in Epidemiology, FF); the Swedish Research Council through the Swedish Initiative for Research on Microdata in the Social And Medical Sciences (SIMSAM) framework (grant No 340-20135867, CA); and the West China Hospital, Sichuan University (1.3.5 Project for Disciplines of Excellence, grant No ZYJC18010, HS). This funding source had no role in study design, data collection, data analysis, data interpretation, writing of the scientific article, or the decision to submit the paper for publication.

Competing interests: All authors have completed the ICMJE uniform disclosure form at www.icmje.org/coi disclosure.pdf and declare: the study was supported by Grant of Excellence, Icelandic Research Fund (grant No 163362-051, UAV), and European Research Council (ERC) consolidator grant (StressGene, grant No 726413, UAV); the Karolinska Institutet (Senior Researcher Award and Strategic Research Area in Epidemiology, FF); the Swedish Research Council through the Swedish Initiative for Research on Microdata in the Social And Medical Sciences (SIMSAM) framework (grant No 340 2013-5867, CA); and the West China Hospital, Sichuan University (1.3.5 Project for Disciplines of Excellence, grant No ZYJC18010, $\mathrm{HS})$; no financial relationships with any organisation that might have an interest in the submitted work in the previous three years; no other relationships or activities that could appear to have influenced the submitted work. 
Ethical approval: This study was approved by the regional ethics review board in Stockholm, Sweden (Dnr 2013/862-31/5).

Data sharing: No additional data available.

The study guarantors (HS and UAV) affirm that this manuscript is an honest, accurate, and transparent account of the study being reported; that no important aspects of the study have been omitted; and that any discrepancies from the study as planned (and, if relevant, registered) have been explained.

This is an Open Access article distributed in accordance with the Creative Commons Attribution Non Commercial (CC BY-NC 4.0) license, which permits others to distribute, remix, adapt, build upon this work non-commercially, and license their derivative works on different terms, provided the original work is properly cited and the use is noncommercial. See: http://creativecommons.org/licenses/by-nc/4.0/.

1 Glaser R, Kiecolt-Glaser JK. Stress-induced immune dysfunction: implications for health. Nat Rev Immunol 2005;5:243-51. doi:10.1038/nri1571

2 Curry JM, Hanke ML, Piper MG, et al. Social disruption induces lung inflammation. Brain Behav Immun 2010;24:394-402. doi:10.1016/j.bbi.2009.10.019

3 Segerstrom SC, Miller GE. Psychological stress and the human immune system: a meta-analytic study of 30 years of inquiry. Psychol Bull 2004;130:601-30. doi:10.1037/0033-2909.130.4.601

4 Cohen S, Tyrrell DAJ, Smith AP. Psychological stress and susceptibility to the common cold. N Engl J Med 1991;325:606-12. doi:10.1056/ NEJM199108293250903

5 Cohen S, Doyle WJ, Turner RB, Alper CM, Skoner DP. Emotional style and susceptibility to the common cold. Psychosom Med 2003;65:652-7. doi:10.1097/01.PSY.0000077508.57784.DA

6 Cohen S, Janicki-Deverts D, Doyle WJ, et al. Chronic stress, glucocorticoid receptor resistance, inflammation, and disease risk. Proc Natl Acad Sci U S A 2012;109:5995-9. doi:10.1073/ pnas.1118355109

7 Pedersen AF, Zachariae R, Bovbjerg DH. Psychological stress and antibody response to influenza vaccination: a meta-analysis. Brain Behav Immun 2009;23:427-33. doi:10.1016/j.bbi.2009.01.004

8 Vedhara K, Cox NK, Wilcock GK, et al. Chronic stress in elderly carers of dementia patients and antibody response to influenza vaccination'. Lancet 1999;353:627-31. doi:10.1016/S01406736(98)06098-X

9 Glaser R, Kiecolt-Glaser JK, Bonneau RH, Malarkey W, Kennedy S, Hughes J. Stress-induced modulation of the immune response to recombinant hepatitis B vaccine. Psychosom Med 1992;54:22-9. doi:10.1097/00006842-199201000-00005

10 Bond E, Lu D, Herweijer E, et al. Sexually transmitted infections after bereavement - a population-based cohort study. BMC Infect Dis 2016;16:419. doi:10.1186/s12879-016-1705-x

11 WHO. The ICD-10 classification of mental and behavioral disorders: Clinical description and diagnostic guidelines (ICD-10). World Health Organization, 1992

12 Passos IC, Vasconcelos-Moreno MP, Costa LG, et al. Inflammatory markers in post-traumatic stress disorder: a systematic review, metaanalysis, and meta-regression. Lancet Psychiatry 2015;2:1002-12. doi:10.1016/S2215-0366(15)00309-0

13 Uddin M, Aiello AE, Wildman DE, et al. Epigenetic and immune function profiles associated with posttraumatic stress disorder. Proc Natl Acad Sci U S A 2010;107:9470-5. doi:10.1073/pnas.0910794107

14 Speer K, Upton D, Semple S, McKune A. Systemic low-grade inflammation in post-traumatic stress disorder: a systematic review. J Inflamm Res 2018;11:111-21. doi:10.2147/JIR.S155903

15 Song H, Fang F, Tomasson G, et al. Association of StressRelated Disorders With Subsequent Autoimmune Disease. JAMA 2018;319:2388-400. doi:10.1001/jama.2018.7028

16 Jiang T, Farkas DK, Ahern TP, Lash TL, Sørensen HT, Gradus IL. Posttraumatic Stress Disorder and Incident Infections: A Nationwide Cohort Study. Epidemiology 2019;30:911-7.

17 D'Onofrio BM, Lahey BB, Turkheimer E, Lichtenstein P. Critical need for family-based, quasi-experimental designs in integrating genetic and social science research. Am J Public Health 2013;103(Suppl 1):S46-55. doi:10.2105/AJPH.2013.301252

18 Ekbom A. The Swedish Multi-generation Register. Methods Mol Biol 2011;675:215-20. doi:10.1007/978-1-59745-423-0_10

19 Harvey AG, Bryant RA. The relationship between acute stress disorder and posttraumatic stress disorder: a 2-year prospective evaluation. J Consult Clin Psychol 1999;67:985-8. doi:10.1037/0022 006X.67.6.985

20 Sharma T, Guski LS, Freund N, Gøtzsche PC. Suicidality and aggression during antidepressant treatment: systematic review and meta-analyses based on clinical study reports. BMJ 2016;352:165. doi:10.1136/bmj.i65

21 Lagerberg T, Molero Y, D’Onofrio BM, et al. Antidepressant prescription patterns and CNS polypharmacy with antidepressants among children, adolescents, and young adults: a population-based study in Sweden. Eur Child Adolesc Psychiatry 2019;28:1137-45. doi:10.1007/s00787-018-01269-2

22 Schäfer I, Hopchet M, Vandamme N, et al. Trauma and trauma care in Europe. Eur J Psychotraumatol 2018;9:1556553. doi:10.1080/200 08198.2018.1556553

23 Baldwin DS, Anderson IM, Nutt DJ, et al. Evidence-based pharmacological treatment of anxiety disorders, post-traumatic stress disorder and obsessive-compulsive disorder: a revision of the 2005 guidelines from the British Association for Psychopharmacology. J Psychopharmacol 2014;28:403-39. doi:10.1177/0269881114525674

24 Ursano RJ, Bell C, Eth S, et al, Work Group on ASD and PTSD, Steering Committee on Practice Guidelines. Practice guideline for the treatment of patients with acute stress disorder and posttraumatic stress disorder. Am J Psychiatry 2004;161(Suppl):3-31

25 Carta MG, Balestrieri M, Murru A, Hardoy MC. Adjustment Disorder: epidemiology, diagnosis and treatment. Clin Pract Epidemiol Ment Health 2009;5:15. doi:10.1186/1745-0179-5-15

26 Gros DF, Price M, Magruder KM, Frueh BC. Symptom overlap in posttraumatic stress disorder and major depression. Psychiatry Res 2012;196:267-70. doi:10.1016/j.psychres.2011.10.022

27 Charlson ME, Pompei P, Ales KL, MacKenzie CR. A new method of classifying prognostic comorbidity in longitudinal studies: development and validation. J Chronic Dis 1987;40:373-83. doi:10.1016/0021-9681(87)90171-8

28 Tutarel O, Alonso-Gonzalez R, Montanaro C, et al. Infective endocarditis in adults with congenital heart disease remains a lethal disease. Heart 2018;104:161-5. doi:10.1136/ heartjnl-2017-311650

29 Harrell FE. Regression modeling strategies, with applications to linear models, survival analysis and logistic regression. Springer-Verlag, 2001.

30 Altman DG, Bland JM. Interaction revisited: the difference between two estimates. BMJ 2003;326:219. doi:10.1136/ bmj.326.7382.219

31 Cohen S, Doyle WJ, Turner R, Alper CM, Skoner DP. Sociability and susceptibility to the common cold. Psychol Sci 2003;14:389-95. doi:10.1111/1467-9280.01452

32 Pedersen A, Zachariae R, Bovbjerg DH. Influence of psychological stress on upper respiratory infection--a meta-analysis of prospective studies. Psychosom Med 2010;72:823-32. doi:10.1097/ PSY.0b013e3181f1d003

33 Ojard C, Donnelly JP, Safford MM, Griffin R, Wang HE. Psychosocial stress as a risk factor for sepsis: a population-based cohort study. Psychosom Med 2015;77:93-100. doi:10.1097/ PSY.0000000000000120

34 van de Beek D, Brouwer M, Hasbun R, Koedel U, Whitney CG, Wijdicks E. Community-acquired bacterial meningitis. Nat Rev Dis Primers 2016;2:16074. doi:10.1038/nrdp.2016.74

35 Adriani KS, Brouwer MC, van de Beek D. Risk factors for communityacquired bacterial meningitis in adults. Neth / Med 2015;73:53-60.

36 Tavaré A, O'Flynn N. Recognition, diagnosis, and early management of sepsis: NICE guideline. BrJ Gen Pract 2017;67:185-6. doi:10.3399/bjgp17X690401

37 Rudd KE, Kissoon N, Limmathurotsakul D, et al. The global burden of sepsis: barriers and potential solutions. Crit Care 2018;22:232. doi:10.1186/s13054-018-2157-z

38 Collaborators GBDM GBD 2016 Meningitis Collaborators. Global, regional, and national burden of meningitis, 1990-2016: a systematic analysis for the Global Burden of Disease Study 2016. Lancet Neurol 2018;17:1061-82. doi:10.1016/S14744422(18)30387-9

39 Frans O, Rimmö PA, Aberg L, Fredrikson M. Trauma exposure and post-traumatic stress disorder in the general population. Acta Psychiatr Scand 2005;111:291-9. doi:10.1111/j.16000447.2004.00463.x

40 Bailey M, Engler H, Hunzeker J, Sheridan JF. The hypothalamic-pituitary-adrenal axis and viral infection. Viral Immunol 2003:16:141-57. doi:10.1089/088282403322017884

41 Silverman MN, Pearce BD, Biron CA, Miller AH. Immune modulation of the hypothalamic-pituitary-adrenal (HPA) axis during viral infection. Viral Immunol 2005;18:41-78. doi:10.1089/vim.2005.18.41

42 Lionakis MS, Kontoyiannis DP. Glucocorticoids and invasive fungal infections. Lancet 2003;362:1828-38. doi:10.1016/S01406736(03)14904-5

43 Aucott IN. Glucocorticoids and infection. Endocrinol Metab Clin North Am 1994;23:655-70. doi:10.1016/S0889-8529(18)30091-4

44 Janicki-Deverts D, Cohen S, Turner RB, Doyle WJ. Basal salivary cortisol secretion and susceptibility to upper respiratory infection. Brain Behav Immun 2016;53:255-61. doi:10.1016/j.bbi.2016.01.013

45 Edwards S, Hucklebridge F, Clow A, Evans P. Components of the diurnal cortisol cycle in relation to upper respiratory symptoms and perceived stress. Psychosom Med 2003;65:320-7. doi:10.1097/01. PSY.0000033123.70631.8E 
46 Biddie SC, Conway-Campbell BL, Lightman SL. Dynamic regulation of glucocorticoid signalling in health and disease. Rheumatology (Oxford) 2012:51:403-12. doi:10.1093/rheumatology/ker215

47 Johnson RR, Prentice TW, Bridegam P, et al. Social stress alters the severity and onset of the chronic phase of Theiler's virus infection. J Neuroimmunol 2006;175:39-51. doi:10.1016/j. ineuroim.2006.02.014

48 Mandell W, Vlahov D, Latkin C, Oziemkowska M, Cohn S. Correlates of needle sharing among injection drug users. Am I Public Health 1994;84:920-3. doi:10.2105/AJPH.84.6.920

49 Sopori M. Effects of cigarette smoke on the immune system. Nat Rev Immunol 2002:2:372-7. doi:10.1038/nri803

50 Danese A, Caspi A, Williams B, et al. Biological embedding of stress through inflammation processes in childhood. Mol Psychiatry 2011;16:244-6. doi:10.1038/mp.2010.5

51 Baumeister D, Akhtar R, Ciufolini S, Pariante CM, Mondelli V. Childhood trauma and adulthood inflammation: a meta-analysis of peripheral C-reactive protein, interleukin-6 and tumour necrosis factor-a. Mol Psychiatry 2016;21:642-9. doi:10.1038/mp.2015.67

52 De Bellis MD, Zisk A. The biological effects of childhood trauma. Child Adolesc Psychiatr Clin N Am 2014;23:185-222, vii. doi:10.1016/j. chc.2014.01.002

53 Kennedy B, Chen R, Valdimarsdóttir U, Montgomery S, Fang F, Fall K. Childhood Bereavement and Lower Stress Resilience in Late Adolescence. J Adolesc Health 2018;63:108-14. doi:10.1016/j. jadohealth.2018.02.002

54 Mehta D, Klengel T, Conneely KN, et al. Childhood maltreatment is associated with distinct genomic and epigenetic profiles in posttraumatic stress disorder. Proc Natl Acad Sci U S A 2013;110:8302-7. doi:10.1073/pnas.1217750110

Supplementary information: Supplementary tables 1-7 and figures 1 and 2 